\title{
Directivity Enhancement in Non-Reciprocal Bandpass Filters using an Evolutionary Algorithm
}

This paper was downloaded from TechRxiv (https://www.techrxiv.org).

\section{LICENSE}

CC BY 4.0

SUBMISSION DATE / POSTED DATE

$06-12-2021 / 10-12-2021$

\section{CITATION}

Dutta, Prantik; Gande, Arun; Ram, Gopi (2021): Directivity Enhancement in Non-Reciprocal Bandpass Filters using an Evolutionary Algorithm. TechRxiv. Preprint. https://doi.org/10.36227/techrxiv.17129423.v1

DOI

10.36227/techrxiv.17129423.v1 


\title{
Directivity Enhancement in Non-Reciprocal Bandpass Filters using an Evolutionary Algorithm
}

\author{
Prantik Dutta, Student Member, IEEE, Gande Arun Kumar, Senior Member, IEEE \\ and Gopi Ram, Senior Member, IEEE
}

\begin{abstract}
In this letter, a non-reciprocal filter with enhanced directivity is analyzed methodically and the filter parameters are optimized using an evolutionary algorithm. The return loss, insertion loss, and isolation characteristics of the filter exhibit a trade-off that makes manual tuning a trial-and-error method. The veracity of the numerical modeling is conformed by designing a $150 \mathrm{MHz}$ lumped element non-reciprocal bandpass filter based on the parameters extracted using an evolutionary algorithm based particle swarm optimization (PSO). The simulated and measured results comply well with the modeling and the results exhibit maximum directivity of $28.2 \mathrm{~dB}$ without degradation in insertion loss $(1.1 \mathrm{~dB})$ and return loss $(16.2 \mathrm{~dB})$ within the passband. The algorithm can be utilized in designing non-reciprocal filters having different center frequencies and bandwidths.
\end{abstract}

Index Terms-Bandpass filter (BPF), isolator, non-reciprocal bandpass filters (NR-BPF), particle swarm optimization (PSO), time-varying transmission line (TVTL), varactor.

\section{INTRODUCTION}

$\mathbf{N}$ ON-RECIPROCITY, using time-varying transmission lines (TVTL), has recently gained laurels in the design of non-reciprocal devices such as circulators, isolators, gyrators, etc., which traditionally relied on bulky and expensive ferrite components for their operations. [1]-[3]. Lately, the concept of TVTL has documented its proficiency in the design of nonreciprocal bandpass filters (NR-BPF).

The first fabricated lumped realization of NR-BPF [4] achieves non-reciprocity at $200 \mathrm{MHz}$ with manual optimization to choose the parameter values. Further, manual optimization has been carried out in the lumped realization of NR-BPF with tunable center frequency [5] and distributed realization of [6]. However, manual optimization results in a degraded insertion loss (IL) and return loss (RL) within the passbands. Subsequent NR-BPF realizations [7]-[9], using similar trends of manual optimization and parametric variation, have further upgraded the design and methodologies to create a trade-off between the non-reciprocity and other parameters like IL and $\mathrm{RL}$ in the passband.

In this paper, the underlying phenomenon behind the distribution of RF signal power among the harmonics, generated due to the modulation of the time-varying resonators, is tabularly illustrated to comprehend the phenomenon. Consequently, the need for an optimization algorithm is judiciously explained, followed by PSO algorithm to obtain the parameter values to achieve the best possible isolation between the forward and reverse paths while preserving the IL and RL in the passband. The numerical model, designed at $150 \mathrm{MHz}$,

Manuscript submitted on August 24th, 2021, revised on October 28, 2021. Prantik Dutta, Gande Arun Kumar and Gopi Ram are with the Department of Electronics and Communication Engineering, National Institute of Technology, Warangal, Telangana, India. e-mail: g.arun@nitw.ac.in.

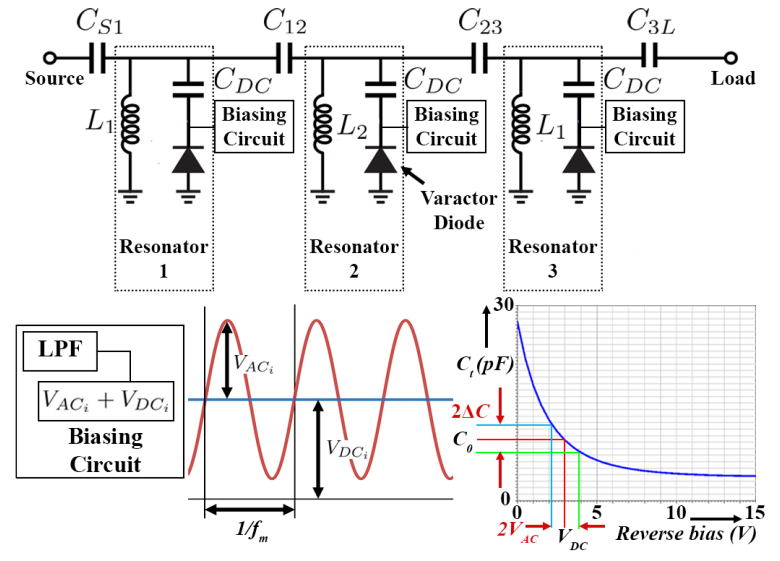

Fig. 1. Schematic of a $3^{r d}$ order NR-BPF (top), the biasing circuit to the varactors and the corresponding generation of time-varying capacitance with the aid of modulation signal having a fixed DC offset.

has been simulated and fabricated using lumped components and the measured results comply well with the modeling and the simulated results.

\section{THE NON-RECIPROCAL PHENOMENON AND THE SCOPE OF OPTIMIZATION}

A typical $3^{\text {rd }}$ order NR-BPF, shown in Fig. 1, consists of space-separated time-varying resonators coupled by immittance inverters (indicated by $C_{S 1}, C_{12}, C_{23}$, and $C_{3 L}$ in the figure). The sinusoidal signals applied to the time-varying capacitors (varactor diodes) in each of the resonators have (a) a DC offset bias to fix the nominal capacitance value $\left(C_{0}\right)$ and (b) an $\mathrm{AC}$ bias, characterized by a constant frequency $\left(f_{m}\right)$ for all the resonators to manifest the deviation in the capacitance value $(\Delta C)$ along with an incremental phase angle between the resonators $(\Delta \phi)$. The parameters $f_{m}$ together with $\Delta \phi$ and the modulation index $\left(\zeta=\Delta C / C_{0}\right)$ hold the key to the manifestation of non-reciprocity at the center frequency $\left(f_{0}\right)$ [4].

The evolution of non-reciprocity lies in the non-uniform distribution of RF power among the generated intermodulation (IM) products in the forward and reverse directions, illustrated in [10]. This entails proper engineering of the circuit parameters to direct the energy flow in the requisite direction to obtain the best possible result both in terms of isolation as well as IL and RL in the passband.

Table I illustrates the numerical simulation results of fractional power conversion among the fundamental and the harmonics of a NR-BPF. The tabular data is obtained after converting the spectral admittance matrix in [11] into an effective spectral $S$-parameter matrix. Tables I(a) and I(b) represent the reflected and transmitted power conversion among the 
TABLE I

Fractional POWER CONVERSiON AMONG THE Fundamental AND IM PRODUCTS.

\begin{tabular}{|c|c|c|c|}
\hline$\left|\boldsymbol{S}_{\mathbf{1 1}}\right|^{\mathbf{2}}$ & $\mathbf{- 1}$ & $\mathbf{0}$ & $\mathbf{1}$ \\
\hline $\mathbf{- 1}$ & 0.338 & ${ }^{\boldsymbol{x}} 0.067$ & 0.067 \\
\hline $\mathbf{0}$ & 0.235 & 0.024 & 0.31 \\
\hline $\mathbf{1}$ & 0.049 & ${ }^{\boldsymbol{x}} 0.034$ & 0.147 \\
\hline
\end{tabular}

(a)

\begin{tabular}{|c|c|c|c|}
\hline$\left|S_{22}\right|^{2}$ & -1 & o & 1 \\
\hline-1 & 0.398 & $\checkmark_{0.257}$ & 0.058 \\
\hline 0 & 0.038 & $\checkmark_{0.018}$ & 0.028 \\
\hline 1 & 0.042 & $\checkmark_{0.253}$ & 0.209 \\
\hline
\end{tabular}

(c)

\begin{tabular}{|c|c|c|c|}
\hline$\left|S_{21}\right|^{2}$ & -1 & o & 1 \\
\hline-1 & 0.224 & 0.021 & 0.009 \\
\hline o & $\begin{array}{ll}x^{x} & 0.036\end{array}$ & $\checkmark^{\prime} 0.821$ & $x^{x} 0.052$ \\
\hline 1 & 0.006 & 0.029 & 0.303 \\
\hline
\end{tabular}

(b)

\begin{tabular}{|c|c|c|c|}
\hline$\left|\boldsymbol{S}_{\mathbf{1 2}}\right|^{\mathbf{2}}$ & $\mathbf{- 1}$ & $\mathbf{0}$ & $\mathbf{1}$ \\
\hline $\mathbf{- 1}$ & 0.116 & 0.159 & 0.177 \\
\hline $\mathbf{0}$ & $\boldsymbol{X}_{0.121}$ & 0.008 & $\boldsymbol{X}^{0.185}$ \\
\hline $\mathbf{1}$ & 0.125 & 0.182 & 0.169 \\
\hline
\end{tabular}

(d) harmonics in the forward direction, respectively, while tables $\mathrm{I}(\mathrm{c})$ and $\mathrm{I}(\mathrm{d})$ represent the reflected and transmitted power conversion among the harmonics in the backward direction, respectively. For instance, the second column of the first row in table I(a) (marked by $\boldsymbol{X}$ ) represents the fraction of power reflected from the first harmonic at the lower side $\left(f_{0}-f_{m}\right)$ to the center frequency $\left(f_{0}\right)$. Similarly, the second column of the second row in table I(b) (marked by $\checkmark$ ) represents the transmission at the center frequency. The symbols $(\boldsymbol{V})$ and $(\boldsymbol{X})$ denotes the desired and undesired components, respectively. Thus, this table enhances the comprehension of the dispersion of the spectral energy both in the forward and reverse direction, which in turn manifests the non-reciprocal phenomenon.

Enhancing the IL in the passband would require increasing the power transmission at the center frequency (cell marked by $\checkmark$ ) in table $\mathrm{I}(\mathrm{b})$ by simultaneously reducing the power converted from the center frequency to the harmonics at the load end (cells marked by $\boldsymbol{X}$ ) in table I(b) and substantially minimizing the RL at the center frequency (marked by gray cell) by reducing the reflected power from the harmonics back to the center frequency at the source end (cells marked by $\boldsymbol{X}$ ) in table I(a). On the other hand, to achieve non-reciprocity at the center frequency, in addition to the fractional reflected power at the fundamental alone, the fractional power reflected from the harmonics to the center frequency (cells marked by $\checkmark)$ in table I(c) should also be enhanced, thereby increasing the total IL in the passband (marked by gray cell) in table I(d); herein lies the scope of optimization. Manual optimization arrives at a crude approximation of the relation between the circuit parameters and the filter characteristics. Another drawback lies in initializing two of the three parameters $(\zeta$, $f_{m}$ and $\Delta \phi$ ) with some arbitrary values to sweep the other parameter at the first iteration. Any erroneous prediction of the initial values would end up in a choice of parameter values far from the global convergence point. Apart from the dubiety in the veracity of this parametric study to search for the best possible result, the technique is arduous and its inefficiency in handling constrained optimization problems happens to be a significant setback. Contrarily, the deployment of an optimization algorithm would consider all the circuit parameters simultaneously, which collectively would strive to obtain the best isolation, apart from maintaining an optimum $\mathrm{IL}$ and RL within the passband. The tables serve as an aid to the optimization algorithm.

\section{PSO ALGORITHM}

Particle Swarm Optimization is one of the metaheuristic algorithms to optimize non-linear continuous functions. The concept of this algorithm has been inspired by swarm intelligence often observed in the social behaviour of a flock of birds or a school of fishes [12]. The population of solutions is termed as swarm which is composed of a number of agents known as particles. The particles are initialized with a random set of solutions which then collectively hover over the problem space, the speed of which depends on a velocity parameter assigned to each particle. The best positions of each particle are tracked in each iteration. Based on these memories, the individual velocities and hence their positions are updated at each iteration. Eventually, the algorithm converges to the best result by the collective participation of the individual particles and fetches the values of the best combination of the parameters.

In this work, PSO algorithm is utilized to extract the optimizing parameter values to obtain the optimal non-reciprocal result in a $3^{\text {rd }}$ order $150 \mathrm{MHz}$ BPF having a bandwidth of 15 MHz. The first step requires the parameters $\zeta, f_{m}$, and $\Delta \phi$ with random values within a given range. The next crucial step lies in exercising a constraint on IL and RL at the center frequency $\left(f_{0}\right)$, considering $n$ harmonics above and below $f_{0}$, and is given in equation (1) as the cost function $(C F)$ shown at the bottom, where, the acronyms $F R, F T, B R$ and $B T$ denote reflection in the forward direction (table $\mathrm{I}(\mathrm{a})$ ), transmission in the forward direction (table $\mathrm{I}(\mathrm{b})$ ), reflection in the backward direction (table $\mathrm{I}(\mathrm{c})$ ) and transmission in the backward direction (table $I(d)$ ), respectively. The variables $\left(F R_{\text {sum }}, B T_{\text {sum }}, F T_{\text {sum }}\right.$ and $\left.B R_{\text {sum }}\right)$ are responsible for summing up the energy contents in the respective harmonics and are given by

$$
\begin{aligned}
F R_{\text {sum }} & =F R_{\text {sum }}+w * F R(m, 0) \\
B T_{\text {sum }} & =B T_{\text {sum }}+w * B T(0, m) \\
F T_{\text {sum }} & =F T_{\text {sum }}+w * F T(0, m)+1 / F T(0,0) \\
B R_{\text {sum }} & =B R_{\text {sum }}+w * B R(m, 0)+B R(0,0)
\end{aligned}
$$

The appearance of the letter ' $a$ ' in equation (1) is a bit intriguing; it is an arbitrary number chosen to have a value much greater than the minimum value of $C F$ that can be obtained. If the conditions are not satisfied, then there is a sudden surge in the deterioration of the value of $C F$ owing to the large value of ' $a$ ', and hence, the position and global best of the algorithm are maintained at the last best values and the algorithm continues its search for the optimal minimum in the right direction. The variable ' $m$ ' in equation (2) varies in the range of $m \leq|n|$ excluding $n=0$ and the variable

$$
C F= \begin{cases}F R_{\text {sum }}+B T_{\text {sum }}+F T_{\text {sum }}+B R_{\text {sum }}, & \text { if } \mathrm{IL} \leq 1 \mathrm{~dB} \& \mathrm{RL} \geq 15 \mathrm{~dB} \\ F R_{\text {sum }}+B T_{\text {sum }}+F T_{\text {sum }}+B R_{\text {sum }}+a, & \text { otherwise }\end{cases}
$$




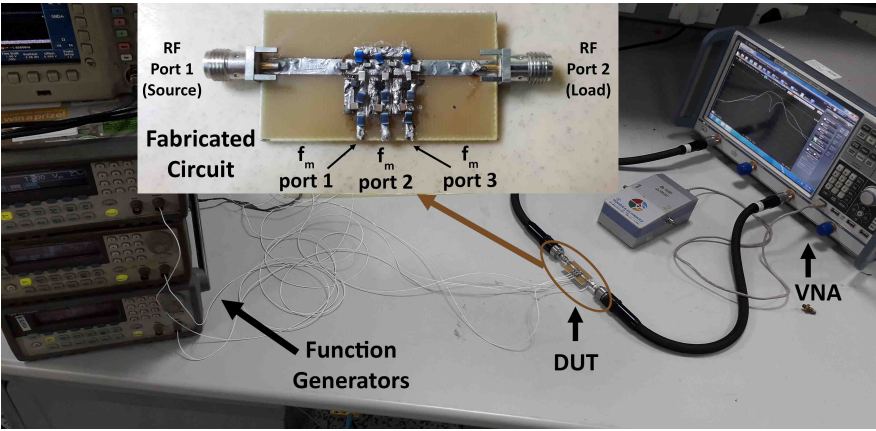

Fig. 2. Experimental setup of the $3^{r d}$ order NR-BPF and the fabricated circuit (inset).

' $w$ ', denoting the associated weight, is given by $w=1 / m$. In this algorithm, to deal exclusively with the spectral contents having the maximum amount of power, the value of $n$ is set to 2 . The numbers within the brackets $(i, j)$ represent the energy translation from the $i^{\text {th }}$ harmonic to the $j^{\text {th }}$ harmonic. For instance, $F T(0,-1)$ represents the energy translation from $f_{0}$ to $f_{0}-f_{m}$ (marked by $\boldsymbol{X}$ ) in the first column of table $\mathrm{I}(\mathrm{b})$. The weight $(w)$ is designed in such a way so as to commensurate the energy content with the degree of the harmonics. This deliberately promotes the algorithm to emphasize more on the harmonics nearer to the center frequency possessing more spectral energy. For the specified band of harmonic content denoted by ' $n$ ', the algorithm calculates the weighted sum of the desired and undesired components in one single loop. It is worthwhile mentioning that the undesired components are specified as reciprocals to facilitate the algorithm to converge to an optimal minimum. On an interesting note, numerical simulations reveal that the algorithm converges to a better result if different modulation indices are applied on each varactor. The algorithm successfully converges after 300 iterations with a population size of 100 . The cognitive and social parameter constants in the velocity and position equations are judiciously chosen as 1.5 [13].

\section{EXPERIMENTAL VALIDATION AND DISCUSSION}

The parameter values extracted using the PSO algorithm $\left(\zeta_{1}=1.49, \zeta_{2}=0.8 \zeta_{3}=0.63, f_{m}=12 \mathrm{MHz}\right.$ and $\left.\Delta \phi=54^{\circ}\right)$ are used to modulate the time-varying capacitors (realized using SMV1236 varactors) in each of the resonators. The circuit parameter values of Fig. 1 are $C_{S 1}=C_{3 L}=6.2$ $\mathrm{pF}, C_{12}=C_{23}=1.8 \mathrm{pF}, L_{1}=L_{3}=82 \mathrm{nH}, L_{2}=100$ $\mathrm{nH}$ and $C_{D C}=82 \mathrm{pF}$. The $\mathrm{AC}$ and $\mathrm{DC}$ biasing values $\left(V_{D C_{1}}=V_{D C_{3}}=2.73 \mathrm{~V}, V_{D C_{2}}=2.04 \mathrm{~V}, V_{A C_{1}}=0.28\right.$ $\mathrm{V}, V_{A C_{2}}=0.25 \mathrm{~V}$ and $V_{A C_{3}}=0.5 \mathrm{~V}$ ), chosen from a piecewise linear region of the varactor characteristic curve using the extracted $\zeta$ values, are applied through a LPF circuit. The circuit is simulated using Cadence AWR software and fabricated on a FR4 substrate, shown in inset of Fig. 2.

Almost comparable correlation of the simulated and measured results with the numerical modeling as shown in Fig. 3(a)-(d) vouches for the accuracy. A slight reduction in bandwidth is observed in the simulation and measurement owing to the usage of standard values of the lumped components and the piecewise linear approximation in the varactor characteristics, which results in a slight deviation of $\Delta \mathrm{C}$ values and hence, the

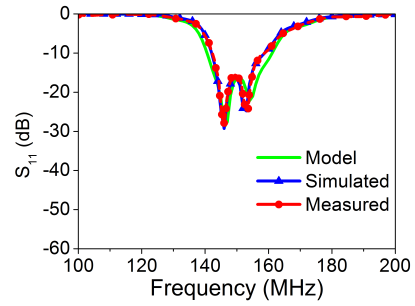

(a) $S_{11}$

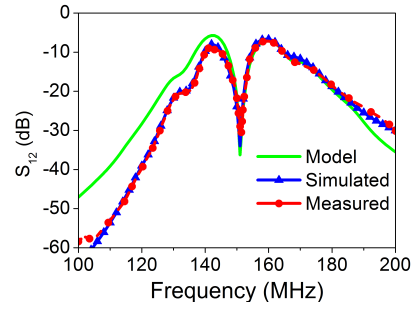

(c) $S_{12}$

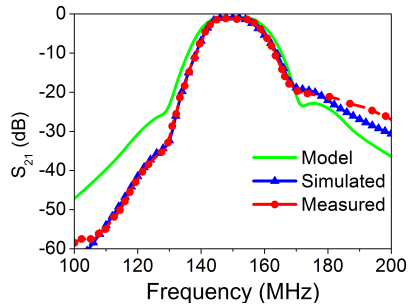

(b) $S_{21}$

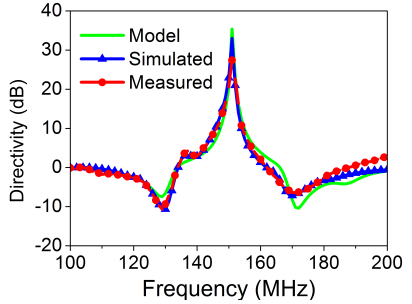

(d) Directivity $\left(S_{21}-S_{12}\right)$
Fig. 3. Comparison of the numerical, simulation and experimental results for (a) input reflection $\left(S_{11}\right)$, (b) forward transmission $\left(S_{21}\right)$, (c) reverse isolation $\left(S_{12}\right)$ and (d) directivity.

TABLE II

COMPARISON WITH OTHER WORKS IN THE LITERATURE

\begin{tabular}{|c||c||c||c||c||c|}
\hline & Technology & $f_{0}(\mathbf{G H z})$ & IL & RL & D \\
\hline \hline$[4]$ & Lumped & 0.19 & 1.5 & 15 & 20.2 \\
\hline$[5]$ & Lumped & 0.14 & 3.7 & $>14$ & 52.8 \\
\hline This work & Lumped & 0.15 & 1.1 & 16.2 & 28.6 \\
\hline
\end{tabular}

IL, RL, and Directivity (D) are in dB scale.

$\zeta$ values. The IL at the center frequency has slightly degraded in the case of circuit simulation $(0.96 \mathrm{~dB})$ and measurement $(1.1 \mathrm{~dB})$ as compared to the numerical result $(0.85 \mathrm{~dB})$ owing to the resistive losses in the varactor. A comparison table with other works in the lumped domain is shown in Table II. The minimum passband IL is $1.1 \mathrm{~dB}$, which is, by far, the best IL achieved so far. A directivity of $28.6 \mathrm{~dB}$ is achieved in the measured results. Even though the directivity is $52.8 \mathrm{~dB}$ in [5], it suffers an IL of $3.7 \mathrm{~dB}$ which not only nullifies the competency of the power amplifier in the transmitter section, it also degrades the noise figure if implemented in the receiver section. The measured RL of the prototype NRBPF is greater than $16 \mathrm{~dB}$, thereby ensuring good matching with the source/load. It is also observed in the simulation and measurement results that constraining the minimum value of the RL to $20 \mathrm{~dB}$ results in further lowering of the bandwidth and a significant drop in the directivity.

\section{CONClusion}

In this paper, PSO algorithm is deployed for the first time to extract the circuit parameters of a $150 \mathrm{MHz}$ NR-BPF using lumped components. Contrary to the manual optimization adapted so far in the literature, which ends up with a rudimentary approximation of the filter parameters, the PSO algorithm searches for the best possible combination of the parameters to obtain the finest result in terms of isolation between the forward and reverse paths, without degrading the IL and RL in the passband; thereby expounding its efficacy in this domain. The algorithm possesses the potential to be exploited to obtain the best possible parametric values for any desired non-reciprocal filter configurations. 


\section{REFERENCES}

[1] C. E. Fay and R. L. Comstock, "Operation of the ferrite junction circulator," IEEE Trans. Microw. Theory Techn., vol. 13, no. 1, pp. 1527, 1965.

[2] J. D. Adam, L. E. Davis, G. F. Dionne, E. F. Schloemann, and S. N Stitzer, "Ferrite devices and materials," IEEE Trans. Microw. Theory Techn., vol. 50, no. 3, pp. 721-737, 2002.

[3] F. A. Ghaffar, J. R. Bray, M. Vaseem, L. Roy, and A. Shamim, "Theory and design of tunable full-mode and half-mode ferrite waveguide isolators," IEEE Trans. on Magnetics, vol. 55, no. 8, pp. 1-8, 2019.

[4] X. Wu, X. Liu, M. D. Hickle, D. Peroulis, J. S. Gómez-Díaz, and A. Álvarez Melcón, "Isolating bandpass filters using time-modulated resonators," IEEE Trans. Microw. Theory Techn., vol. 67, no. 6, pp. $2331-2345,2019$

[5] D. Simpson and D. Psychogiou, "Magnet-less non-reciprocal bandpass filters with tunable center frequency," in 2019 49th Eur. Microw. Conf., 2019, pp. 460-463.

[6] A. Alvarez-Melcon, X. Wu, J. Zang, X. Liu, and J. S. Gomez-Diaz, "Coupling matrix representation of nonreciprocal filters based on timemodulated resonators," IEEE Trans. Microw. Theory Techn., vol. 67, no. 12, pp. 4751-4763, 2019.

[7] X. Wu, M. Nafe, A. . Melcón, J. S. Gómez-Díaz, and X. Liu, "Frequency tunable non-reciprocal bandpass filter using time-modulated microstrip $\lambda_{g} / 2$ resonators," IEEE Trans. Circuits Syst. II Exp. Briefs, pp. 1-1, 2020.

[8] X. Wu, M. Nafe, A. A. Melcón, J. Sebastián Gómez-Díaz, and X. Liu, "A non-reciprocal microstrip bandpass filter based on spatio-temporal modulation," in 2019 IEEE MTT-S Int. Microw. Symp., 2019, pp. 9-12.

[9] X. Wu, M. Nafe, and X. Liu, "Non-reciprocal 2nd-order bandpass filter by using time-modulated microstrip quarter-wavelength resonators," in 2019 Int. Conf. on Microw. and Milli. Wave Tech. (ICMMT), 2019, pp. $1-3$.

[10] P. Dutta, G. Arun Kumar, G. Ram, and D. Suneel Varma, "Spatiotemporal non-reciprocal filters: Theoretical concepts and literature review," IEEE Microwave Magazine, (in press).

[11] P. Dutta, G. Arun Kumar, G. Ram, and D. Suneel Varma, "Magnetless 3-pole non-reciprocal bandpass filter: Numerical analysis with matlab simulation," in 2021 International Conference on Computing, Соттиnication, and Intelligent Systems (ICCCIS), 2021, pp. 810-813.

[12] J. Kennedy and R. Eberhart, "Particle swarm optimization," in Proc. of ICNN'95 - Int. Conf. on Neural Networks, vol. 4, 1995, pp. 1942-1948.

[13] A. R. Jordehi, "A review on constraint handling strategies in particle swarm optimisation," Neural Comp. and Appl., vol. 26, pp. 1265-1275, 2015. 